\title{
Visco-resistive shear wave dissipation in magnetic X-points
}

\author{
G. Fruit and I. J. D. Craig
}

University of Waikato, New Zealand

e-mail: fruit@waikato.ac.nz

Received 19 September 2005 / Accepted 3 November 2005

\section{ABSTRACT}

We consider the viscous and resistive dissipation of perpendicularly polarized shear waves propagating within a planar magnetic $X$-point. To highlight the role played by the two-dimensional geometry, the damping of travelling Alfvèn waves that propagate within an unbounded, but non-orthogonal $X$-point topology is analyzed. It is shown that the separatrix geometry affects both the dissipation time and the visco-resistive scaling of the energy decay. Our main focus, however, is on developing a theoretical description of standing wave dissipation for orthogonal, line-tied $X$-points. A combination of numerical and analytic treatments confirms that phase mixing provides a very effective mechanism for dissipating the wave energy. We show that wave decay comprises two main phases, an initial rapid decay followed by slower eigenmode evolution, both of which are only weakly dependent on the visco-resistive damping coefficients.

Key words. Sun: magnetic fields - magnetohydrodynamics (MHD) - plasmas - Sun: flares - Sun: corona

\section{Introduction}

The heating of the solar corona remains a major issue in space physics. It is strongly believed that the magnetic structure of the corona plays a dominant role in this phenomenon. In particular, the magnetic field lines are frozen in the photosphere and their footpoints are thus constrained to follow the random photospheric motions. If these displacements are slow the field lines become twisted and magnetic energy is gradually stored in the coronal arcade until its release via magnetic reconnection (Ruzmaikin \& Berger 1998). On the contrary, if footpoint motions are fast, they generate MHD waves that propagate along open or closed field lines (Hollweg 1987). Due to the inhomogeneities in the solar plasma, wave energy can be transfered into heat via phase mixing (Heyvaerts \& Priest 1983; Craig \& Fruit 2005) or resonant absorption (Ionson 1978; Tirry et al. 1997; Fruit et al. 2002).

Reconnection models have been extensively studied in the literature (see e.g. Priest \& Forbes 2000) but a recurring difficulty stems from the low resistivities of typical coronal plasmas. The fact that the dimensionless resistivity $\eta$ is an inverse Lundquist number of order $10^{-14}$ (Spitzer 1962), makes it very difficult to construct mechanisms that achieve sufficiently rapid energy release. Even when fast reconnection is formally demonstrated (e.g. Craig \& McClymont 1991, 1993 and Hassam 1992), the huge field localizations required to make the mechanism effective $\left(\ell \leq \eta^{1 / 2}\right)$ can be undone by a variety of effects. For instance, the back pressure of axial field components is known to inhibit the rapid collapse of planar magnetic $X$-points.

On the other hand, viscous dissipation of Alfvèn waves, whose effects are not burdened by such weak damping coefficients, should be more robust $(v \gg \eta)$. Hollweg (1986) has already emphasized theoretically that viscosity could be important in a wide variety of coronal processes. In the case of a magnetic loop whose footpoints are anchored by the photosphere, Alfvèn waves bounce back and forth at a frequency that depends on the length and strength of the field line. Large spatial gradients across the field lines result from the difference in these eigenfrequencies. This leads to enhanced viscous or resistive dissipation on time scales of the order of $(v+\eta)^{-1 / 3}$ as discussed in Sect. 5.2. Phase mixing thus provides an efficient mechanism for energy dissipation.

Up to now the phase mixing of Alfvèn waves has been analyzed mainly in one-dimensional geometries. Typically the magnetic field is inhomogeneous in the direction perpendicular to the straight magnetic field lines (see Heyvaerts \& Priest 1983; Hood et al. 1997a,b). A two-dimensional configuration was studied by Ruderman et al. (1998) with the assumption that the inhomogeneity along the field lines was much weaker than across the field lines. Ideal wave propagation in the vicinity of null points has recently been considered by McLaughlin $\& \operatorname{Hood}(2004,2005)$ but relatively few studies have included a detailed analysis of visco-resistive wave dissipation (see Hassam \& Lambert 1996).

Our present aim is to study the visco-resistive damping by phase mixing in the vicinity of $2 \mathrm{D}$ null points. In particular, we extend the analysis of Craig \& Fruit (2005) which applies in a simple 1D neutral sheet. After introducing the MHD system in Sect. 2, we discuss the influence of the $X$-point (separatrix) geometry in the damping of freely propagating waves (Sect. 3). In Sect. 4 we turn to the study of an orthogonal, line-tied $X$-point. We find that although the initial damping phase is in good agreement with the previous phase mixing 
studies, the presence of a null point in the background magnetic field leads to interesting new features in the later decay (see Sect. 5). In particular, fast initial dissipation may be followed by oscillatory exponential decay and by a more slowly decreasing asymptotic tail, due to the emergence of viscous quasi-eigenmodes or self-similar modes in the vicinity of the null (see Sect. 5.3). A key result is that the later stages of the decay depend only weakly on the viscous and resistive coefficients. Although, in the present initial value problem, the bulk of the wave energy is lost in the initial stages of the damping, the asymptotic phases could govern the response of a "driven" system in which energy is constantly supplied - for instance by buffeting of the footpoints (Ruderman et al. 1997). This intriguing possibility is left to a future investigation.

\section{The MHD equations}

In what follows we consider the motions of a viscous and resistive plasma of uniform density embedded in a planar magnetic $X$-point, scaled with respect to the reference parameters

$B_{0}=10^{2} \mathrm{G}, \quad l_{0}=10^{9.5} \mathrm{~cm}$

$n_{0}=10^{9} \mathrm{~cm}^{-3}, \quad v_{\mathrm{A}}=10^{9} \mathrm{~cm} \mathrm{~s}^{-1}$.

In these units time is measured in units of the Alfvén time $\tau_{\mathrm{A}}=$ $l_{0} / v_{\mathrm{A}}$ which is typically a few seconds for coronal parameters.

We assume that the equilibrium magnetic field, defined by the flux function

$\psi_{\mathrm{E}}=\frac{1}{2}\left(y^{2}-\alpha^{2} x^{2}\right)$,

is perturbed by shear wave disturbances polarized perpendicular to the plane. We are interested in how rapidly these disturbances are viscously and resistively damped, and to what extent the background $X$-point geometry affects the dissipation. In this connection note that the background field $\boldsymbol{B}_{\mathrm{E}}=(y, \alpha x)$ has orthogonal separatrices only for $\alpha=1$ and that, as $\alpha \rightarrow 0$, the field degenerates to the one-dimensional form $\boldsymbol{B}_{\mathrm{E}}=y \hat{\boldsymbol{x}}$ considered by Craig \& Fruit (2005).

Our aim is to contrast wave propagation in an unbounded plasma with dissipation in a "closed" $X$-point whose field lines are anchored by a highly conducting rigid boundary. In either case we are interested in the evolution of the axial disturbance fields, namely $\boldsymbol{v}=W(x, y, t) \hat{\boldsymbol{z}}$ and $\boldsymbol{B}=Z(x, y, t) \hat{z}$. These fields are governed by the non-dimensional momentum and induction equations

$$
\begin{aligned}
& \frac{\partial W}{\partial t}=\left(\boldsymbol{v}_{\mathrm{A}} \cdot \nabla\right) Z+v \nabla^{2} W \\
& \frac{\partial Z}{\partial t}=\left(\boldsymbol{v}_{\mathrm{A}} \cdot \nabla\right) W+\eta \nabla^{2} Z
\end{aligned}
$$

with $\boldsymbol{v}_{A} \equiv \boldsymbol{B}_{E}=(y, \alpha x)$ is the normalized Alfven velocity.

Note that we have assumed Laplacian forms for the dimensionless viscosity $v$ and resistivity $\eta$. Although viscosity can generally be expected to dominate resistivity for collisional plasmas, we should remember that, for strong magnetic fields, the viscosity is a highly non-isotropic tensor and that the hydrodynamic form we adopt is accurate only in a small region surrounding the $X$-point null (Braginskii 1965). In view of these uncertainties we investigate system (2)-(3) for small but arbitrary $v$ and $\eta$.

Before examining wave damping in a line-tied $X$-point geometry, we first consider the propagation of travelling waves in an unbounded plasma.

\section{Damping of freely propagating waves}

\subsection{Effect of $X$-point geometry}

We begin by generalizing the analysis of Craig \& Fruit (2005) who considered the visco-resistive phase mixing of a perpendicularly polarized shear wave propagating in an unbounded one-dimensional magnetic field $\boldsymbol{B}_{\mathrm{E}}=y \hat{\boldsymbol{x}}$. We still introduce the Elsasser variables $Z \pm W$ but retain the two dimensionality of the background field by taking $\alpha>0$. This allows us, not only to highlight new effects that derive from relaxing the assumption of a strictly one-dimensional background field, but also to provide a physical context for the line-tied standing wave calculations of Sect. 4.

Introducing the variables $g(x, y, t), h(x, y, t)$ defined by

$g=Z+W \quad h=Z-W$

we find that system (2)-(3) reduces to

$\frac{\partial g}{\partial t}=+y \frac{\partial g}{\partial x}+\alpha^{2} x \frac{\partial g}{\partial y}+\epsilon_{+} \nabla^{2} g+\epsilon_{-} \nabla^{2} h$

$\frac{\partial \boldsymbol{h}}{\partial t}=-y \frac{\partial h}{\partial x}-\alpha^{2} x \frac{\partial h}{\partial y}+\epsilon_{+} \nabla^{2} h+\epsilon_{-} \nabla^{2} g$

where $\epsilon_{ \pm}=v \pm \eta$.

The system decouples in the case $\eta=v$ and it is possible to determine exact solutions for $g$ and $h$. In particular, free wave solutions can be sought of the form

$\left(\begin{array}{l}g \\ h\end{array}\right)=\exp (i m(t) x+i k(t) y)\left(\begin{array}{l}A^{+}(t) \\ A^{-}(t)\end{array}\right)$

We find that

$\dot{A}=-\epsilon_{+}\left(m^{2}+k^{2}\right) A, \quad \dot{m}= \pm \alpha^{2} k, \quad \dot{k}= \pm m$,

where $A$ stands for either $A^{ \pm}$. Since $g$ and $h$ have very similar damping rates we can simplify the analysis by concentrating on the evolution of $g(x, y, t)$, formally setting $h=0$. In this case, taking positive signs in the previous expressions, we find that the wavenumbers of $g$ grow according to

$m=m_{0} \cosh (\alpha t)+\alpha k_{0} \sinh (\alpha t)$

$k=k_{0} \cosh (\alpha t)+\frac{m_{0}}{\alpha} \sinh (\alpha t)$

where $m_{0}$ and $k_{0}$ are initial values.

In the case of an orthogonal $X$-point $(\alpha=1)$, we see that there is comparable exponential growth in both wavenumbers $m$ and $k$, corresponding to a near isotropic localization in the disturbance field. For a one-dimensional $X$-point however, with $\alpha$ arbitrarily small, we obtain a linear growth in $k$ with $m$ constant:

$m=m_{0}, \quad k=k_{0}+m_{0} t, \quad \alpha=0$. 
In all cases the maximum damping rate is attained when the time derivative of $A\left(m^{2}+k^{2}\right)$ in (7) vanishes. We find that the wavenumbers at maximum damping satisfy

$\left(m^{2}+k^{2}\right)^{2} \epsilon_{+}=2\left(1+\alpha^{2}\right) m k$.

Thus, for the one-dimensional background field $\alpha \rightarrow 0, m \rightarrow$ $m_{0}$, the wavenumber $k$ has the amplitude

$k^{3} \simeq \frac{2 m_{0}}{\epsilon_{+}}, \quad \alpha=0$

and this is achieved at the time

$\tau_{0}=\left(\frac{2}{\epsilon_{+} m_{0}^{2}}\right)^{1 / 3}$.

For the orthogonal $X$-point these scalings are changed to

$m^{2} \simeq k^{2} \simeq \frac{1}{\epsilon_{+}}, \quad \alpha=1$,

which leads to

$\tau_{1}=\frac{1}{2 \alpha} \ln \left(\frac{1}{\epsilon_{+}}\right)$

in the case $m_{0} \simeq k_{0} \simeq 1$. It follows that the wavenumbers at maximum dissipation change from an $\epsilon^{1 / 2}$ to an $\epsilon^{1 / 3}$ scaling as the $X$-point field collapses into one-dimensionality.

\subsection{Application to line-tied $X$-points}

The previous analysis shows that the details of the $X$-point geometry can be expected to play a strong role in the damping of the plasma. Although it is not clear a-priori how these results can be applied to a line-tied geometry, we expect that both dissipation timescales and damping rates will be affected. In fact we shall find that the one-dimensional $X$-point model provides a better guide for wave dissipation in the line-tied geometry. This is because the $m=m_{0}$ solution can be interpreted as the fundamental vibrational mode of a one-dimensional, line-tied field of length $\pi / m_{0}$. In this case the field lines are straight but, because the field strength varies linearly with $y$, standing waves of frequency $\omega=m_{0} y$ can develop. Phase mixing leads to the emergence of strong cross-field corrugations which build up in time, and eventually provide strong viscous and resistive damping. In this case solution (7) for the wave amplitude reduces to

$A(t)=A_{0} \exp \left[-\frac{\epsilon_{+}\left(k_{0}+m_{0} t\right)^{3}}{3 m_{0}}\right], \quad \alpha=0$.

We see that the decay is dominated by a negative exponent which grows as $t^{3}$, consistent with (9).

Now consider the case of a line-tied orthogonal $X$-point. The field lines vary in length and are now curved, but wave motions are still controlled by the directional derivative $\boldsymbol{v}_{\mathrm{A}} \cdot \nabla$ acting along the field. As we show below, standing waves develop on each field line, leading, at least initially, to strong cross-field corrugations and rapid damping. This initial phase follows very closely the $-t^{3}$ exponential decay predicted by the one-dimensional result (10).

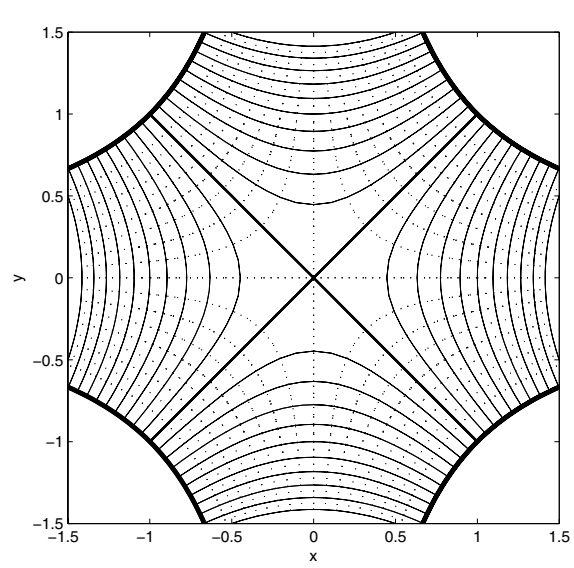

Fig. 1. The X-point background equilibrium using the flux function $\psi_{\mathrm{E}}=(1 / 2)\left(y^{2}-x^{2}\right)$. The magnetic field lines (solid lines) are tied to the very thick hyperbolic contours $\varphi= \pm \phi_{0}= \pm 1$. Dotted lines are the orthogonal curves to the field lines $(\varphi=$ const.).

\section{The orthogonal line-tied $X$-point}

We now specialize to the case of shear wave damping in an orthogonal $X$-point plasma where $\alpha=1$. The evolution Eqs. (2)-(3) take the explicit forms

$\frac{\partial W}{\partial t}=y \frac{\partial Z}{\partial x}+x \frac{\partial Z}{\partial y}+v \nabla^{2} W$
$\frac{\partial Z}{\partial t}=y \frac{\partial W}{\partial x}+x \frac{\partial W}{\partial y}+\eta \nabla^{2} Z$

and these are simplified by working in a curvilinear frame defined by the equilibrium field lines, namely

$\psi=\frac{1}{2}\left(y^{2}-x^{2}\right) \quad \phi=x y$.

The $\varphi=$ const. and $\psi=$ const. are two sets of hyperbolic orthogonal curves shown in Fig. 1. In this new coordinate system Eqs. (11)-(12) become

$\frac{\partial W}{\partial t}=2 r^{2} \frac{\partial Z}{\partial \varphi}+2 v r^{2} \nabla^{2} W$

$\frac{\partial Z}{\partial t}=2 r^{2} \frac{\partial W}{\partial \varphi}+2 \eta r^{2} \nabla^{2} Z$

with $r^{2}=\sqrt{\varphi^{2}+\psi^{2}}=x^{2}+y^{2}$

and $\nabla^{2}=\frac{\partial^{2}}{\partial \varphi^{2}}+\frac{\partial^{2}}{\partial \psi^{2}}$.

The advective term consists only of the derivative along the field line, which simplifies greatly the analysis in the ideal case. In this case $\psi$ is simply a parameter which labels the field lines.

We assume the following boundary and initial conditions. The magnetic field lines are anchored into a rigid boundary $\varphi= \pm \varphi_{0}=$ const. (thick contour in Fig. 1) where the velocity field is assumed to vanish. The shape of this boundary is chosen for mathematical simplicity. In all that follows the shear axial motions are initiated by a given velocity field $W_{0}(x, y)=W(x, y, t=0)$ with no magnetic disturbance. 


\subsection{The ideal solution}

Consider first the simple case where both resistivity and viscosity are turned off. To solve the system (14)-(15) it is convenient to take $\varphi=\psi \sinh (u)$ (Hassam \& Lambert 1996). In these new variables $(\psi, u)$, the ideal system reduces to the classical d'Alembert equations

$$
\begin{aligned}
& \frac{\partial W}{\partial t}=2 \frac{\partial Z}{\partial u} \\
& \frac{\partial Z}{\partial t}=2 \frac{\partial W}{\partial u} .
\end{aligned}
$$

Given the boundary conditions $W\left(\varphi=\varphi_{0}, \psi, t\right)=W(u=$ $\left.u_{0}, \psi, t\right)=0$, we expand the fields in Fourier series

$$
\begin{aligned}
& W(u, \psi, t)=\sum_{n} W_{n}(\psi, t) \sin n k u \\
& Z(u, \psi, t)=\sum_{n} Z_{n}(\psi, t) \cos n k u
\end{aligned}
$$

where

$$
k=\frac{\pi}{u_{0}(\psi)}, \quad \text { and } \quad u_{0}(\psi)=\operatorname{asinh}\left(\frac{\varphi_{0}}{\psi}\right) .
$$

It is now straightforward to derive

$$
\begin{aligned}
& W(u, \psi, t)=\sum_{n} a_{n} \sin \left(n \pi \frac{u}{u_{0}}\right) \cos \left(\omega_{n} t\right) \\
& Z(u, \psi, t)=\sum_{n} a_{n} \cos \left(n \pi \frac{u}{u_{0}}\right) \sin \left(\omega_{n} t\right)
\end{aligned}
$$

where $\omega_{n}=2 n k(\psi)$ and $a_{n}$ is the $n$th Fourier coefficient in the expansion of the initial velocity profile $W(u, \psi, t=0)$.

As expected each field line, being rigidly tied at the end, oscillates back and forth like piano strings. The amplitude of the oscillations is constant - energy in the ideal system is conserved - but the oscillation frequency depends continuously on the field line, via the coordinate $\psi$. As in the one-dimensional model of Sect. 3, the phase between two neighbouring lines increases as time goes on, leading to linearly growing crossgradients - the $\partial / \partial \psi$ terms in the Laplacian. It is these burgeoning gradients that eventually lead to strong visco-resistive dissipation.

\section{Visco-resistive dissipation}

\subsection{Numerical example of energy damping}

In order to gain insight into the problem, the system (14)-(15) is solved numerically with the initial velocity profile vanishing away from the neutral point

$W(\varphi, \psi, t=0)=\sin \left(\pi \frac{\varphi}{\varphi_{0}}\right) \psi \exp \left(-\frac{\psi^{2}}{2 L^{2}}\right)$.

The numerical procedure uses an explicit predictor-corrector scheme based on second order differences with Dirichlet (Neumann) boundary conditions for the velocity field (magnetic field) on $\varphi= \pm \varphi_{0}$. Both fields are also put to zero on the limiting field line $\psi= \pm \psi_{0}-$ a condition which is expected to play a minor role if $\psi_{0}$ is chosen much greater than $L$. In pratice we choose $\psi_{0}=1$ and $L=1 / \sqrt{20} \sim 0.22$.

Figure 2 shows qualitatively the time evolution of the shear disturbances in the $(x, y)$ plane for $v=10^{-3}$ and $\eta=0$. Only the velocity field $W(x, y, t)$ is represented using a gray scale for the amplitude. The initial gaussian shape is lost after the first few Alfvèn times, the shear waves being rapidly structured by the background magnetic field. The increasing phase shift between all these vibrations creates corrugations across the field lines of smaller and smaller wavelength. Once they reach an appropriate spatial scale, the diffusion acts very efficiently for removing the bulk of the initial energy. As seen on the central panel of Fig. 3, the global energy stored in the system falls in 5 orders of magnitude during the first 10 Alfvèn time. However, the damping of the energy eventually slows down and the later evolution is manifestly different. As shown later, this phase is characterized by quite complicated oscillatory modes. To clarify the analysis the kinetic (dashed) or magnetic (solid) energy decay represented in Fig. 3 may be divided into three stages:

1. An initial evolution characterized by a smooth and very fast decay of the energy. This lasts until 10 Alfvèn times and also corresponds to the building-up of the corrugations (see the three above panels of Fig. 3). Equipartition of kinetic and magnetic energies is well represented during this stage.

2. An oscillatory and exponentially damped phase follows, revealing most likely the presence of a global eigenmode. Energy equipartition is still preserved. As seen on the lower panels of Fig. 3 the corrugations have almost disappeared and only a very localized perturbation persists along the separatrix $\psi=0$.

3. An asymptotic tail of slow damping takes place at roughly 25 Alfvèn times. A clear separation of kinetic and magnetic energy is now visible. The dominant magnetic disturbance is even more localized along the separatrix than in the previous stage (bottom right panel).

In the following analysis, the first two stages will be studied in more detail. The next section provides an approximate analytical solution for the initial stage which is consistent with all the previous studies of phase mixing. Section 5.3 gives an interpretation of the second phase using approximate scaling law arguments. In view of the small amount of energy left in the system at the beginning of the third stage, it does not seem worthwhile presenting a detailed analysis of the late decay. We remark however, that a self-similar mode was analyzed in the 1D study of Craig \& Fruit (2005) which closely resembles the present asymptotic phase.

\subsection{Analytical solution for the initial evolution}

First of all, we emphasize that the background geometry is simple enough to allow an exact transformation of the original 2D problem into an a-priori simpler 1D problem. By using the same change of variables as in Sect. 4, the dissipative system (14)-(15) may be written

$\frac{\partial W}{\partial t}=2 \frac{\partial Z}{\partial u}+2 v \psi \cosh u \frac{\partial^{2} W}{\partial \psi^{2}}$ 

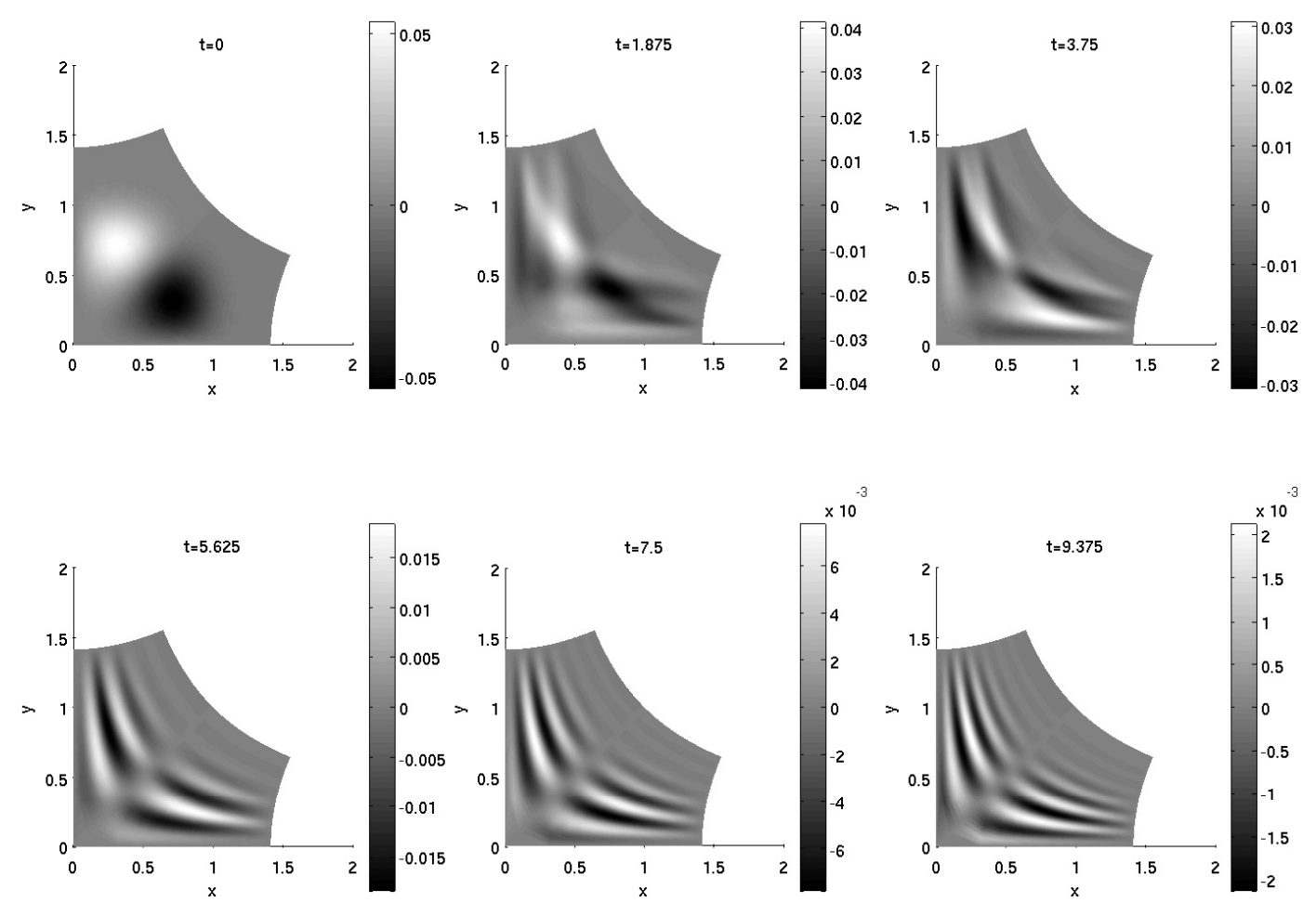

Fig. 2. Temporal evolution of the velocity field in the $(x, y)$-plane (upper right quadrant only) for $v=10^{-3}, \eta=0$ and various times mentioned above the graphs. For clarity, the background X-point has been rotated through $45^{\circ}$ so that the separatrices are aligned to the coordinate axes. A gray scale is used for the amplitude of the velocity field. As time goes on, more and more corrugations are building up along the static magnetic field lines until the transversal wavelength becomes of the order of a diffusion length when efficient damping occurs. Only a small perturbation concentrated along the separatrix remains thereafter.

$$
\frac{\partial Z}{\partial t}=2 \frac{\partial W}{\partial u}+2 \eta \psi \cosh u \frac{\partial^{2} Z}{\partial \psi^{2}}
$$

where

$u=\operatorname{asinh}(\varphi / \psi)$.

Although, to simplify the writing, we have retained only the dominant cross field gradients in the Laplacian operator, the other terms may be written in a similar fashion.

As before we expand $W$ and $Z$ in Fourier series (Eqs. (18)-(19)) and make use of the cosine series representation

$\cosh u=\frac{c_{0}}{2}+\sum_{n=1}^{\infty} c_{n} \cos (n k u)$

with

$c_{n}(\psi)=2 \frac{\sinh u_{0}}{u_{0}} \frac{(-1)^{n}}{1+(n k)^{2}}$.

Identifying each Fourier harmonic, we get after some algebra

$\dot{W}_{n}=-2 n k Z_{n}+v \psi \sum_{p=1}^{\infty}\left(c_{|n-p|}-c_{n+p}\right) W_{p}^{\prime \prime}$

$\dot{Z}_{n}=+2 n k W_{n}+\eta \psi \sum_{p=1}^{\infty}\left(c_{|n-p|}+c_{n+p}\right) Z_{p}^{\prime \prime}$

where the dot denotes the time derivative and the prime corresponds to the $\psi$-derivative. The system (25)-(26) is now spatially $1 \mathrm{D}$ as it depends only on $\psi$. If we keep all the terms in the
Laplacian, the transformation $2 \mathrm{D} \rightarrow 1 \mathrm{D}$ remains formally exact. But the two-dimensionality of the problem is not lost since the different harmonics along the curved magnetic field lines are now, contrary to the true $1 \mathrm{D}$ problem, coupled together.

Without any further approximation, system (25)-(26) is as difficult to solve as the original system (23)-(24). However, since the magnitude of the coupling coefficients $c_{n}$ will decrease rapidly with $n$, at least for a smooth initial profile, it is possible to base an analytic approximation on the fundamental mode, and to neglect the coupling between the higher harmonics. Consequently, we keep only the $p=n=1$ term in the above summations. A more accurate analysis including higher harmonics is possible but it turns out to be unnecessary for the final result (see Fig. 4).

We now derive an analytical approximation for the initial stage of the evolution in the case of small diffusion coefficients. The ideal solution (20)-(21) is assumed to remain valid, at least in its general form, so that the $\psi$-derivative may be written (for $n=1)$

$\frac{\partial W_{1}}{\partial \psi}=a_{1} \pi u \frac{\mathrm{d} k}{\mathrm{~d} \psi} \cos \pi k u \cos 2 k t-2 a_{1} t \frac{\mathrm{d} k}{\mathrm{~d} \psi} \sin \pi k u \sin 2 k t$.

The second term grows linearly in time and thus becomes rapidly dominant over the first term, which can be neglected. To the leading order, the $\psi$-derivative may be replaced by a simple multiplication

$\frac{\partial^{2}}{\partial \psi^{2}} \quad \rightarrow \quad-\left(2 \frac{\mathrm{d} k}{\mathrm{~d} \psi} t\right)^{2} \times$ 

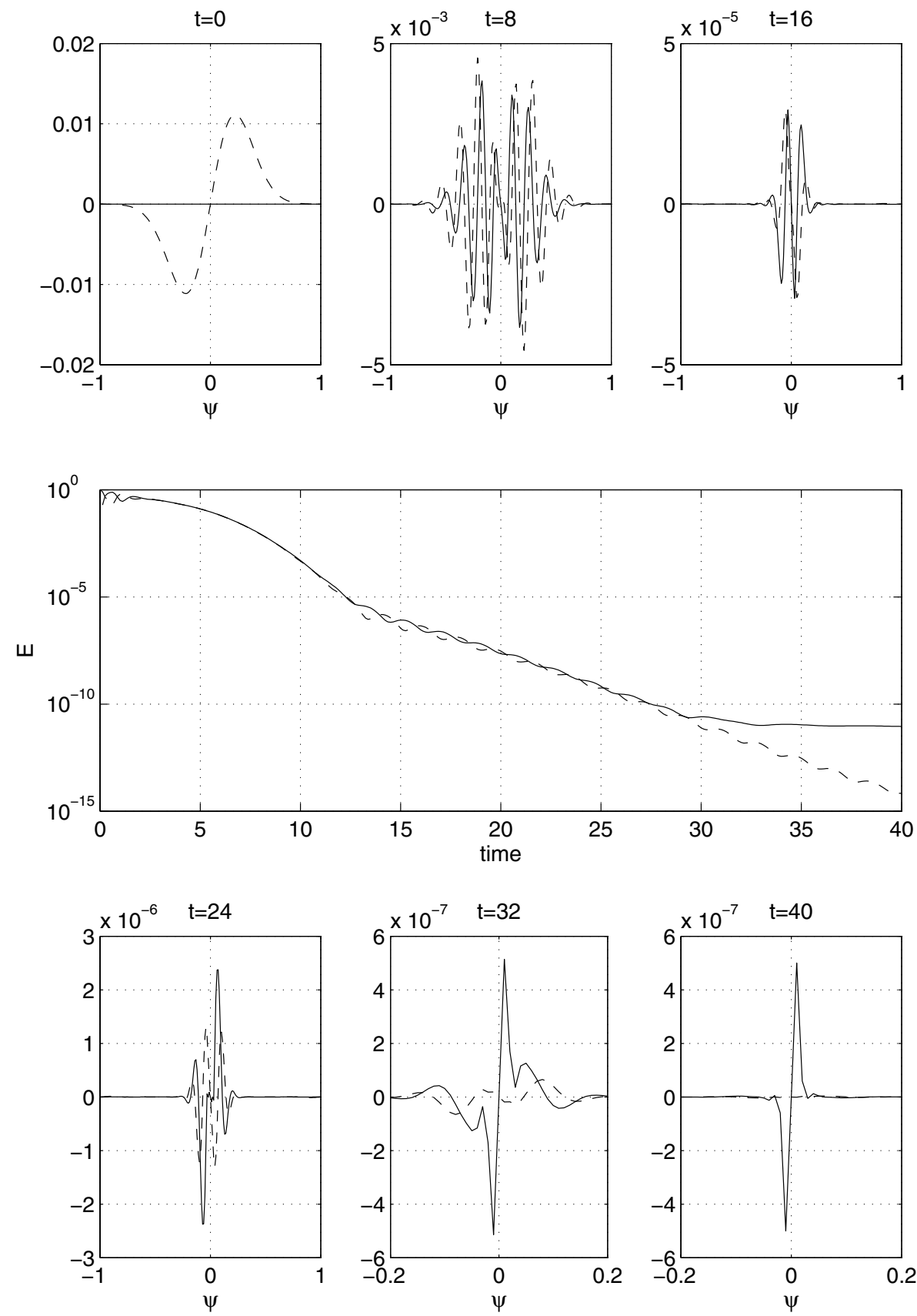

Fig. 3. Viscous dissipation of the total kinetic energy (dashed) and the total magnetic energy (solid) stored in the system for $v=10^{-3}$ and $\eta=0$. Three stages are clearly visible: fast initial decay by phase mixing, exponential damping and very slow decay combined with a separation in the two forms of energy. All around are displayed several snapshots of the velocity field (dashed) and the magnetic field (solid) versus $\psi$ for a given $\varphi=0.5$ at different times mentioned above the graphs. In the last two panels, the $\psi$-axis is rescaled in order to show more clearly the emergence of the self-similar behaviour.

and the system (25)-(26) may be rewritten in the following matrix form

$\frac{\mathrm{d}}{\mathrm{d} t}\left(\begin{array}{l}W_{1} \\ Z_{1}\end{array}\right)=\left(\begin{array}{cc}-v \kappa_{-} t^{2} & -2 k \\ 2 k & \eta \kappa_{+} t^{2}\end{array}\right)\left(\begin{array}{l}W_{1} \\ Z_{1}\end{array}\right)$

with $\kappa_{ \pm}=4 \psi k^{\prime 2}\left(2 c_{0} \pm c_{2}\right)$. Discarding terms of the order of $\eta v$ the eigenvalues of the matrix are

$\lambda_{ \pm}= \pm 2 i k-\frac{v \kappa_{-}+\eta \kappa_{+}}{2} t^{2}$, and as long as the diagonal terms are small compared to the non-diagonal terms, the eigenvectors reduce to $W_{1} \pm i Z_{1}$.

It follows that an approximate solution for the initial evolution is given by

$$
\begin{aligned}
& W_{1}=a_{1} \cos (2 k t) \exp \left(-\frac{\left(v \kappa_{-}+\eta \kappa_{+}\right)}{6} t^{3}\right) \\
& Z_{1}=a_{1} \sin (2 k t) \exp \left(-\frac{\left(v \kappa_{-}+\eta \kappa_{+}\right)}{6} t^{3}\right)
\end{aligned}
$$




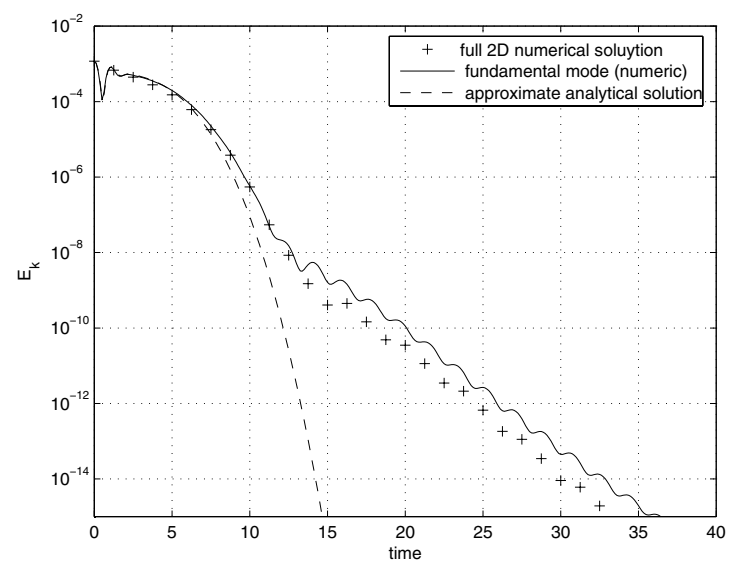

Fig. 4. Comparison between the full numerical solution (cross), the fundamental mode (solid) and the approximate analytical solution (dashed). Global kinetic energy is represented as a function of time and values $v=10^{-3}, \eta=0$ have been chosen.

where $a_{1}$ is the first Fourier coefficient of the initial velocity profile $W(\varphi, \psi, t=0)$. The amplitude of the shear disturbances begins to decrease according to the factor $\exp \left(-\beta t^{3}\right)$ which, as noted in Sect. 3, is a basic characteristic of the phase mixing phenomenon (see also Heyvaerts \& Priest 1983; Craig \& Fruit 2005).

Some insight into the validity of the analytic approximations can be gained from Fig. 4. This represents the time decay of the global kinetic energy for $v=10^{-3}$ and $\eta=0$ using various degrees of approximation: specifically, the crosses correspond to the exact full 2D numerical solution; the solid line gives the 1D numerical solution retaining the fundamental mode only (system (25)-(26) with $n=1, p=1$ ); and the dashed line represents the kinetic energy evaluated from the analytical expression (30)-(31) via a classical quadrature routine. Although these plots represent only the early phases of the evolution, the 1D-fundamental approximation clearly gives a good representation of the full 2D solution. In particular the change of behaviour occurs at the same time and the two tails share the same slope. This suggests that, at least as far as describing the response of the system to the gaussian impulse, it may be unnecessary to model the detailed coupling of the higher harmonics.

We conclude that the cubic law $\exp \left(-\beta t^{3}\right)$ of (30)-(31) describes rather accurately the early damping of the energy. With the large value of $v$ chosen here the matching lasts roughly 6-7 Alfvèn times, during which time most of the initial energy is dissipated. In fact similar behaviour should be expected in every closed system where field lines oscillate at a continuously varying frequency $\omega(\psi)$. The actual variation of the frequency in terms of $\psi$ does not need to be known precisely since it appears in expressions (30)-(31) only through the gradient $\mathrm{d} \omega / \mathrm{d} \psi$.

Later on, the numerical solution tends to decrease more slowly before undergoing a radical change of behaviour after a time $t_{c h}$ which scales as $v^{-1 / 3}$ as seen clearly in Fig. 5. This scaling law - consistent with Eq. (9) - can be inferred from a qualitative argument based on the fact that phase mixing

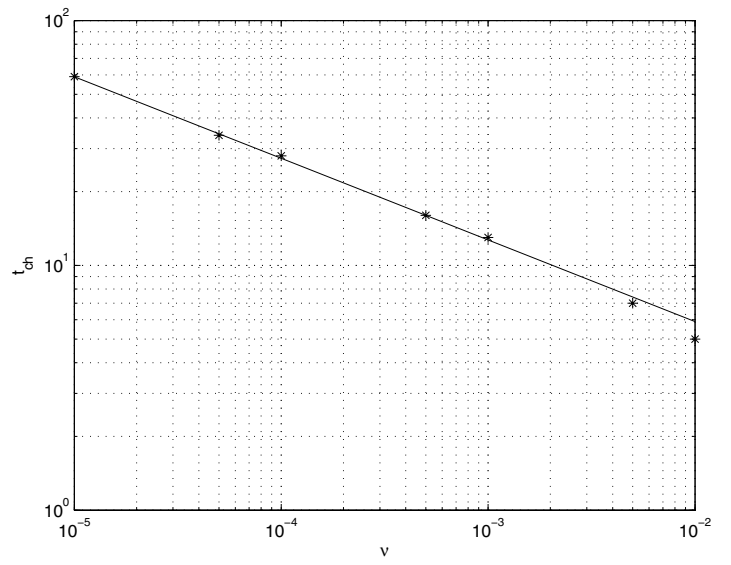

Fig. 5. Logarithmic fit of the time $t_{\mathrm{ch}}$ that separates the fast initial damping phase and the slower exponential decay phase. The stars correspond to the numerical results and the straight line of slope $-1 / 3$ fits the data with a very good agreement.

produces crossfield corrugations of typical length scale $\ell_{\text {id }}$ (Craig \& Fruit 2005). It can be shown from an expansion around a given field line of the phase term $2 k(\psi) t$ that $\ell_{\text {id }}$ diminishes as the inverse of time. Meanwhile the viscosity dissipates the energy over a typical length scale $\ell_{\text {dif }} \propto \sqrt{v t}$ growing as the square root of time. The evolution changes of nature when these two scales become of the same order that is at a time $t_{\mathrm{ch}} \propto v^{-1 / 3}$. This time also corresponds to a maximum viscous dissipation rate since information can diffuse the most efficiently across the tuned undulations of the system.

Once the advective and diffusive length scales are equalized, another viscous phenomenon arises in the system which dramatically affects the damping of the Alfvèn waves. Relatively little energy is involved at this stage, but the details of the wave damping could still be relevant in a driven problem. Accordingly it seems worthwhile to pursuing the possibility of oscillatory damping during the secondary phases of the dissipation.

\subsection{The eigenmode phase}

Recall that the second phase in the evolution of the decay is characterized by exponentially damped oscillations in both the magnetic and velocity fields. This phase is most simply investigated by retaining just the fundamental mode and turning off the resistivity. System (25)-(26) then reduces to

$\dot{W}=-\omega Z+v \frac{\varphi_{0}}{\pi} \frac{\omega^{3}}{1+\omega^{2}} W^{\prime \prime}$

$\dot{Z}=+\omega W$

where $\omega=2 k(\psi)=2 \pi / \operatorname{asinh}\left(\varphi_{0} / \psi\right) \sim 2 \pi / \ln \left(2 \varphi_{0} / \psi\right)$ for small $\psi$. Eliminating $Z$ gives

$\ddot{W}=-\omega^{2} W+\epsilon \frac{\omega^{3}}{1+\omega^{2}} \dot{W}^{\prime \prime}$

where $\epsilon=\varphi_{0} v / \pi$. This equation (34) has no analytical solution despite its relative simplicity. Thus, we restrict the analysis to numerical results and give a heuristic interpretation based on scaling arguments. 


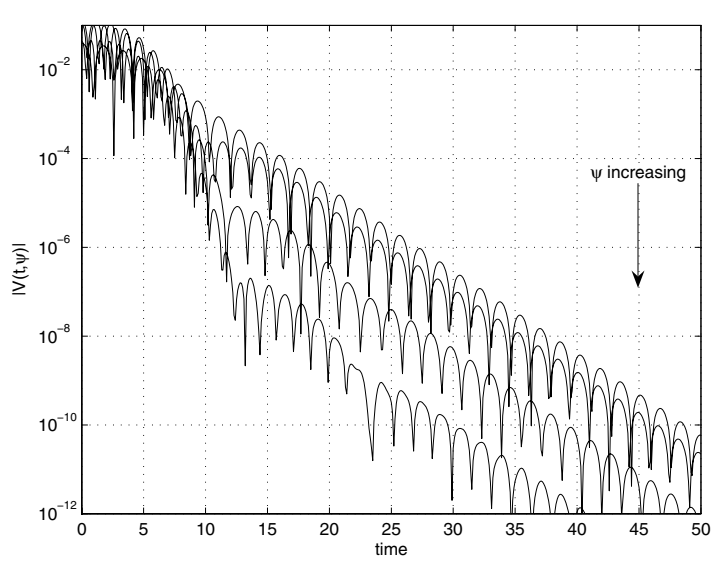

Fig. 6. Plot of the modulus of the velocity field $V(t, \psi)$ versus time for different field lines: from top to bottom $\psi=0.04-0.19-0.34-$ 0.49 . The transition between the two stages in the evolution occurs at different times according to the position from the neutral point. The exponential decay sets up almost immediately close to the separatrix whereas it takes longer further away.

The apparition of a global eigenmode with a unique frequency and a unique damping rate appears peculiar at first sight. In our earlier interpretation, each field line oscillates at its own frequency and the difference between all these frequencies leads to an increasingly corrugated structure. However, the diffusion process eventually limits the build-up in corrugations and, as shown in Fig. 6, slowly orchestrates the corrugations into a single oscillator. Here the velocity field $W(t, \psi)$, solution of (34), is represented as the function of time for various positions $\psi$ in the system. Although the initial decay is pretty much the same for all the chosen positions, the transition towards exponential damping does not occur at the same time: it occurs most rapidly close to the separatrix ( $\psi$ small) but takes longer as $\psi$ increases. But eventually, the whole system seems to oscillate at a unique period $T$ and the energy damps at a single rate, revealing the emergence of a pseudo eigenmode. We use here the term "pseudo" because it is not a true eigenmode of the system. The fact that this oscillatory mode is set up at different times according to the position in the system shows that it cannot be an actual global eigenmode - for there will always be distant field lines which are oscillating independently. Even so, for all practical purposes, we can regard the system as a global oscillator when the bulk of the wave energy has been captured by the outward propagating diffusive influence. The oscillatory mode observed numerically results from a delicate balance of the advective and diffusive terms appearing in (34). It is no longer possible to neglect one of them to simplify the analysis any further. The fact that decay modes of this form were not found in the 1D analysis of Craig \& Fruit (2005) means that the two-dimensionality of the $X$-point must play a key role in the orchestration of the mode.

It is now legitimate to take for the velocity/magnetic field a time dependence of the form $\exp (-\lambda t)$, regarding $\lambda=\alpha+i \Omega$ as effectively independent of $\psi$. Substituting this ansatz in (34) yields

$\lambda^{2} W=-\omega^{2} W+\epsilon \lambda \frac{\omega^{3}}{1+\omega^{2}} W^{\prime \prime}$

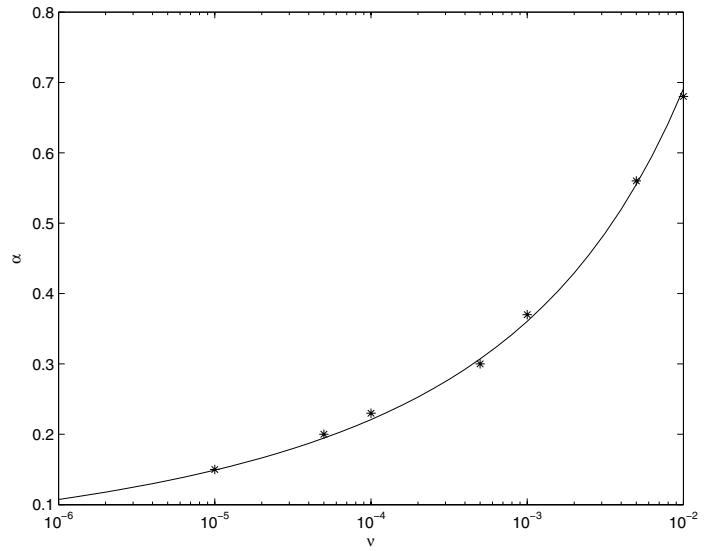

Fig. 7. Rate of exponential damping as a function of the viscosity. The fitting curve is proportional to $\left(\frac{1}{\ln (2 / \sqrt{v})}\right)^{2}$.

We are interested in how $\lambda$ scales with the viscosity parameter $\epsilon \simeq v$. To that end we first note that, since the oscillation frequency $\omega$ varies logarithmically with $\psi$, there must be some field line, say $\psi=\psi^{*}$, which naturally mimics the global oscillation rate of the system. In this case

$\Omega=\omega\left(\psi^{*}\right) \simeq \frac{2 \pi}{\ln \left(2 \varphi_{0} / \psi^{*}\right)}$.

We shall regard $\psi^{*}$ as a delimiter that separates an inner diffusive region from an outer region where, at least for $\psi \gg \psi^{*}$, advection is dominant. On the other hand if the diffusive term is to be comparable with the advective term in (35) the cross field corrugations must scale as $\sqrt{\epsilon}$ (ignoring the weak logarithmic dependencies with $\psi$ of $\omega$ and $\lambda$ ). Identifying the scale of the corrugations with the extent of the inner region defined by $\psi^{*}$ gives a scaling for the global oscillation frequency

$\Omega \simeq \frac{2 \pi}{\ln (2 / \sqrt{\epsilon})}$.

The damping rate $\alpha$ is more difficult to infer but since the outer field oscillations are responsible for building-up the corrugations that initiate the exponential damping it is natural to expect that $\alpha$ is a function of $\Omega$. This suggests that the damping should also have only a weak dependence on viscosity.

This reasoning is reflected by the numerical results displayed in Figs. 7 and 8. Of particular importance is the fact that both the oscillation frequency and the dissipation rate depend very weakly on $v$ : in particular $\alpha$ hardly doubles over three orders of magnitude. This is an important result as it shows that energy can still be effectively dissipated in a very weak dissipative plasma. The linear fit of Fig. 8 confirms the validity of Eq. (36) and a fit of the damping rate $\alpha$ in Fig. 7 suggests the simple following law:

$\alpha \propto \Omega^{2} \propto \frac{1}{[\ln (2 / \sqrt{\epsilon})]^{2}}$.

\section{Conclusion}

We have considered the visco-resistive energy dissipation of shear Alfvén waves within two-dimensional $X$-point magnetic 


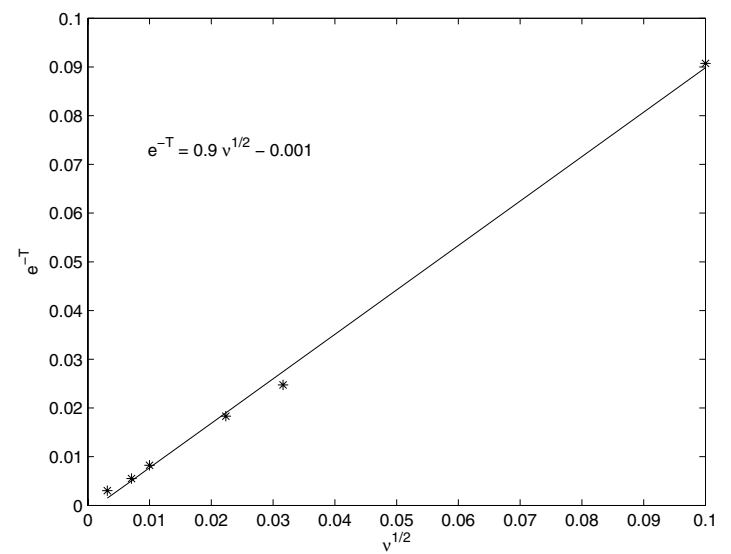

Fig. 8. Period of the oscillations during the second stage. In order to get a linear fit the quantity $\exp (-T)$ is plotted versus $\sqrt{\epsilon}$ (stars). The solid line is a linear fit of the data, showing that the period is proportional to the logarithm of the viscosity.

configurations. The problem is formulated as an initial value problem in which a finite amount of energy is injected into the system and we are interested in how fast this energy is dissipated by phase mixing. It is well known that in a $1 \mathrm{D}$ nonhomogeneous magnetic slab, the wave energy is damped on the timescale $\tau_{m} \simeq(\eta+v)^{-1 / 3}$ (Heyvaerts \& Priest 1983). But the two-dimensionality of the background magnetic field as well as the boundary conditions are likely to affect this scaling law. Indeed it has already been pointed out by Craig \& Fruit (2005), that the presence of a null point dramatically changes the asymptotic nature of the energy decay in 1D slab geometries. It follows that similar behaviour can be expected for the two-dimensional $X$-points considered here.

In Sect. 3 we investigated the problem of free propagation of Alfven waves in an open $X$-point geometry. The scaling of the dissipation rate, and the time of maximum damping, were shown to depend critically on the separatrix angle of the $X$-point. Although the fastest rate was achieved for the orthogonal $X$-point, the quasi one-dimensional configuration was found to provide the best guide to the wave damping in achieved in "closed" line-tied models. The reason stems from the natural development of standing waves in the one-dimensional geometry, which develop into highly corrugated structures due to phase mixing.

Our central results, derived in Sects. 4 and 5, focus on the wave dissipation in orthogonal line-tied $X$-points. The boundary conditions now impose fixed values for the wave number along the field lines, and only one wave number - that associated with cross field stratifications - is allowed to grow in time. Computations of the integrated energy reveal three stages in the wave damping: first, a fast energy decay linked with the build-up of increasingly sharp corrugations across the field lines; second, a slower decay, associated with the emergence of a global eigenmode structure; finally, a very slow asymptotic tail in which the magnetic and kinetic energy are no longer bound together by equipartition. This final stage plays only a minor role in the bulk energetics.

Theoretically, the initial phase can be understood in terms of the balance between the the smoothing effects of visco-resistive diffusion and the building-up of sharp crossfield gradients by phase mixing. We have pointed out that the relatively fast, cubic damping law first proposed by (Heyvaerts $\&$ Priest 1983) provides a reasonably accurate description of the early dissipation stage. In general we can expect the cubic law to account for the bulk of the wave energy dissipation in any closed magnetic configuration which is subject to phase mixing. It is conceivable however, that the later stages of the evolution could become more important in a driven problem where energy is continually injected into the system, say by the continual buffeting of footpoints (see e.g. Ruderman et al. 1997). In this case the weak logarithmic dependence of the damping rate on the dissipation coefficients, as witnessed in the global eigenmode phase, could lead to a quasi-steady regime where the strong visco-resistive dissipation is balanced by a constant supply of energy from the boundaries.

In conclusion we note that a more accurate analysis of the $X$-point damping problem should take into account the tensor form of the viscosity coefficient (Braginskii 1965). Given that the magnetic geometry plays a key role in organizing the wave energy damping for homogeneous dissipation coefficients, it seems natural to expect an even greater sensitivity when the highly anisotropic nature of the viscous tensor is accounted for. The wave damping properties of resistivity when coupled with tensor viscosity are currently being explored.

Acknowledgements. This work was supported by the Marsden Fund (02-UOW-050 MIS).

\section{References}

Berghmans, D., De Bruyne, P., \& Goossens, M. 1996, ApJ, 472, 398 Braginskii, S. I. 1965, Rev. Plasma Phys., 1, 205

Craig, I. J. D., \& McClymont, A. N. 1991, ApJ, 371, 41

Craig, I. J. D., \& McClymont, A. N. 1993, ApJ, 405, 207

Craig, I. J. D. \& Fruit, G. 2005, A\&A, 440, 357

Fruit, G., Louarn, P., Tur, A., \& Le Quéau, D. 2002, JGR, 107, 1411

Hassam, A. B. 1992, ApJ, 399, 159

Hassam, A. B., \& Lambert, R. P. 1996, ApJ, 472, 832

Heyvaerts, J., \& Priest, E. R. 1983, A\&A, 117, 220

Hollweg, J. V. 1986, ApJ, 306, 730

Hollweg, J. V. 1987, ApJ, 320, 875

Hood, A. W., Ireland, J., \& Priest, E. R. 1997, A\&A, 318, 957

Hood, A. W., Gonzalez-Delgado, D., \& Ireland, J. 1997, A\&A, 324, 11

Ionson, J. A. 1987, ApJ, 226, 650

McLaughlin, J. A., \& Hood, A. W. 2004, A\&A, 420, 1129

McLaughlin, J. A., \& Hood, A. W. 2005, A\&A, 435, 313

Parker, E. N. 1997, Sol. Phys., 176, 219

Ruderman, M. S., Goossens, M., Ballester, J. L., \& Oliver, R. 1997, A\&A, 328, 361

Ruderman, M. S., Nakariakov, V. M., \& Roberts, B. 1998, A\&A, 338, 1118

Ruzmaikin, A., \& Berger, M. 1998, A\&A, 337, L9

Priest, E. R., \& Forbes, T. 2000, Magnetic Reconnection (Cambridge University Press)

Spitzer, L. 1962, Physics of fully ionized gases (John Wiley \& Sons)

Tirry, W. J., Berghmans, D., \& Goossens, M. 1997, A\&A, 322, 329 